\title{
Pulmonary cavitation in a patient with coronavirus disease 2019 during lenvatinib treatment for thyroid carcinoma: a case report
}

\author{
Soji Toda^, Ai Matsui, Mio Yasukawa, Daisuke Murayama^, Hiroyuki Iwasaki^ \\ Department of Breast and Endocrine Surgery, Kanagawa Cancer Center, Yokohama, Japan \\ Correspondence to: Soji Toda. Department of Breast and Endocrine Surgery, Kanagawa Cancer Center, Yokohama 241-8515, Japan. \\ Email: s-toda@kcch.jp.
}

\begin{abstract}
Lenvatinib, a multi-tyrosine kinase inhibitor, is used for the treatment of thyroid carcinoma. However, it can cause pneumonia and pulmonary cavitation leading to pneumothorax. The mechanism underlying the occurrence of cavitation and pneumothorax is not well understood. Coronavirus disease 2019 (COVID-19), which is an infectious condition characterized primarily by pneumonia, is sometimes accompanied by pulmonary cavitation. Patients with COVID-19 who present with pulmonary cavitation may have a poor prognosis. In the present case, a patient with papillary thyroid carcinoma presented with multiple pulmonary metastatic tumors that were treated with lenvatinib. After 9 weeks from treatment initiation, he experienced fever and presented with pulmonary consolidation and ground-glass opacity (GGO). Pneumonia improved after the withdrawal of lenvatinib. After 21 weeks from treatment initiation, he developed fever again and the clinical tests led to the diagnosis of COVID-19. Computed tomography (CT) showed new GGO in both sides of the lung. Therefore, the patient was diagnosed with moderate COVID-19. He was treated with dexamethasone plus remdesivir, and GGO due to COVID-19 disappeared. However, the previous pulmonary shadow associated with lenvatinib became a cavitary lesion. The initial CT findings of COVID-19 and pneumonia associated with lenvatinib are similar. Thus, both conditions must be considered for a differential diagnosis in patients presenting with GGO during lenvatinib treatment.
\end{abstract}

Keywords: Lenvatinib; coronavirus disease 2019 (COVID-19); pulmonary cavitation; angiogenesis inhibitor; case report

Submitted Sep 17, 2021. Accepted for publication Nov 23, 2021.

doi: 10.21037/apm-21-2663

View this article at: https://dx.doi.org/10.21037/apm-21-2663

\section{Introduction}

Lenvatinib is a multi-tyrosine kinase inhibitor that suppresses angiogenesis. It is currently used for the treatment of radioactive iodine-refractory differentiated thyroid carcinoma because of its clinical benefits (1). Recent studies have reported a few cases where pulmonary cavitation occurred mainly in patients presenting with pulmonary metastases during lenvatinib treatment (2-4). This may lead to pneumothorax, which prompts the discontinuation of cancer treatment. However, the mechanism underlying the occurrence of pulmonary cavitation and pneumothorax is not well understood.

Recently, coronavirus disease 2019 (COVID-19) has been found to be an important cause of pneumonia. Pulmonary cavitation rarely occurs in patients with COVID-19, and it is associated with a high morbidity and mortality (5-7). The initial imaging findings of pneumonia associated with lenvatinib and COVID-19 are similar. Therefore, clinicians must be aware of the fact that pulmonary cavitation can result from either by COVID-19 or lenvatinib treatment. We present the following case in accordance with the CARE

\footnotetext{
^ ORCID: Soji Toda, 0000-0002-2977-4419; Daisuke Murayama, 0000-0002-1148-4659; Hiroyuki Iwasaki, 0000-0002-9724-8525.
} 

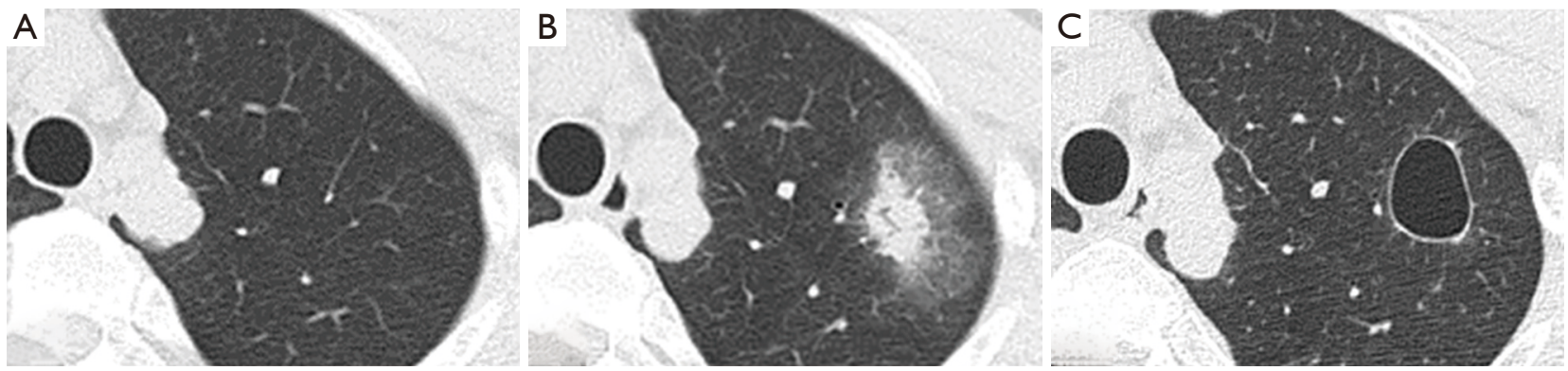

Figure 1 Pneumonia associated with lenvatinib. (A) At 4 months before lenvatinib treatment initiation. (B) At 9 weeks after lenvatinib treatment initiation. (C) At 21 weeks after lenvatinib treatment initiation. The patient presented with COVID-19. CT showed consolidation and GGO in the upper lobe of the left lung (B). After 2 weeks of lenvatinib withdrawal, the fever subsided and the lesion became cavitary (C). COVID-19, coronavirus disease 2019; CT, computed tomography; GGO, ground-glass opacity.
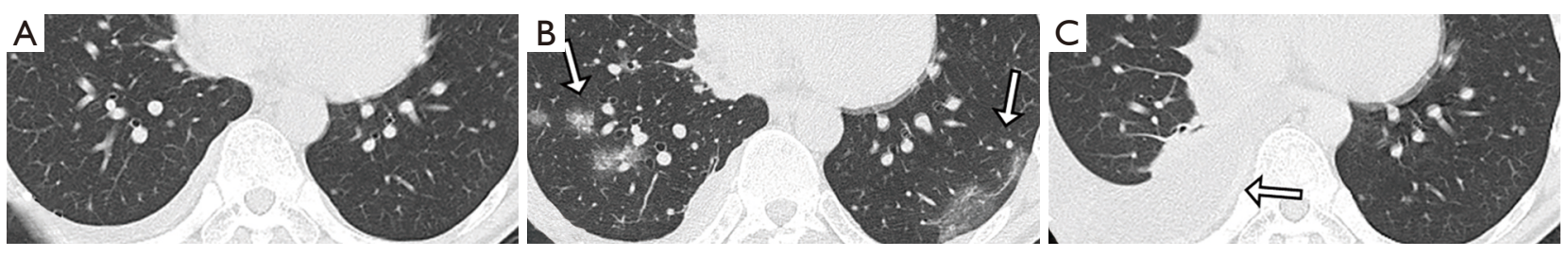

Figure 2 Pneumonia caused by COVID-19. (A) At 9 weeks after lenvatinib treatment initiation. (B) At 21 weeks after lenvatinib treatment initiation. The patient presented with COVID-19. (C) At 31 weeks after lenvatinib treatment initiation. CT showed GGO in both sides of the lung (arrows in B), and the viral antigen and RTPCR tests confirmed COVID-19. After the treatment for COVID-19, GGO disappeared. However, pleural effusion worsened (arrow in C). Thyroid carcinoma progressed after the withdrawal of lenvatinib. COVID-19, coronavirus disease 2019; CT, computed tomography; GGO, ground-glass opacity; RTPCR, reverse transcriptase polymerase chain reaction.

reporting checklist (available at https://apm.amegroups. com/article/view/10.21037/apm-21-2663/rc).

\section{Case presentation}

A 31-year-old man presented to our hospital with a mass in his right neck. His anamnesis revealed Wolff-ParkinsonWhite syndrome and asthma in childhood. He had no family history of cancer. He was diagnosed with papillary thyroid carcinoma (T2N1bM0, the Union for International Cancer Control, eighth edition), and total thyroidectomy, bilateral neck dissection, and upper mediastinal dissection were performed. After 2.5 years of surgeries, he presented with multiple pulmonary metastatic tumors and an elevated blood thyroglobulin level. Radioactive iodine therapy and scintigraphy revealed no uptake in the pulmonary metastatic tumors. After 4 years and 11 months of the surgeries, pulmonary metastasis progressed and pleural effusion appeared. Therefore, lenvatinib treatment was initiated at a reduced dose of $20 \mathrm{mg} /$ day because of the history of WolffParkinson-White syndrome.

The patient developed proteinuria 5 weeks after treatment initiation, and the dose was reduced to $14 \mathrm{mg} /$ day. He had fever at 9 weeks, and computed tomography (CT) showed consolidation and ground-glass opacity (GGO) in the upper lobe of the left lung where no metastasis was observed in a previous CT (Figure 1). The results of severe acute respiratory syndrome coronavirus 2 (SARS-CoV-2) antigen and reverse transcriptase polymerase chain reaction (RTPCR) tests were negative, and the fever disappeared 2 weeks after lenvatinib withdrawal.

At 21 weeks after treatment initiation, he developed fever again and tested positive for the SARS-CoV-2. CT revealed a new GGO in both sides of the lung (Figure 2). The previous pulmonary shadow became a cavitary lesion. Because the patient's oxygen saturation level was $92 \%$ on room air, oxygen therapy via a nasal cannula was initiated. He was diagnosed with moderate COVID-19 based on the 


\begin{tabular}{|c|c|c|c|c|c|c|c|c|c|c|c|c|c|c|c|}
\hline Day of illness & 1 & 2 & 3 & 4 & 5 & 6 & 7 & 8 & 9 & 10 & 11 & 12 & 13 & $\ldots$ & 21 \\
\hline Viral test & & & & + & & & & & & & + & & & & Negative \\
\hline Hospitalization & & & & & & & & & & & & & & & \\
\hline Fever $\left({ }^{\circ} \mathrm{C}\right)$ & + & + & + & 39.0 & 37.0 & 36.8 & 36.9 & 37.1 & 36.9 & 37.0 & 37.4 & & & & \\
\hline Heart rate & & & & 102 & 80 & 84 & 86 & 88 & 90 & 78 & 74 & & & & \\
\hline Oxygen therapy & & & & & & & & & & & & & & & \\
\hline Remdesivir & & & & 200 & 100 & 100 & 100 & 100 & & & & & & & \\
\hline Dexamethasone & & & & 8 & 8 & 8 & 8 & 8 & 4 & 4 & 4 & 4 & 4 & & \\
\hline Heparin calcium & & & & & & & & & & & & & & & \\
\hline
\end{tabular}

Figure 3 Clinical course of COVID-19. The patient was hospitalized on Day 4 of illness. He required oxygen therapy via a nasal cannula for 2 days. Intravenous remdesivir at a dose of $200 \mathrm{mg} /$ day was administered. Then, the dose was reduced to $100 \mathrm{mg} / \mathrm{day}$ for 4 days more. Moreover, the patient received oral dexamethasone at a dose of $8 \mathrm{mg} /$ day for 5 days. Next, the dose was reduced to $4 \mathrm{mg} /$ day for 5 days. Subcutaneous heparin calcium 5,000 units twice daily was administered as a venous thromboembolism prophylaxis treatment. COVID-19, coronavirus disease 2019.

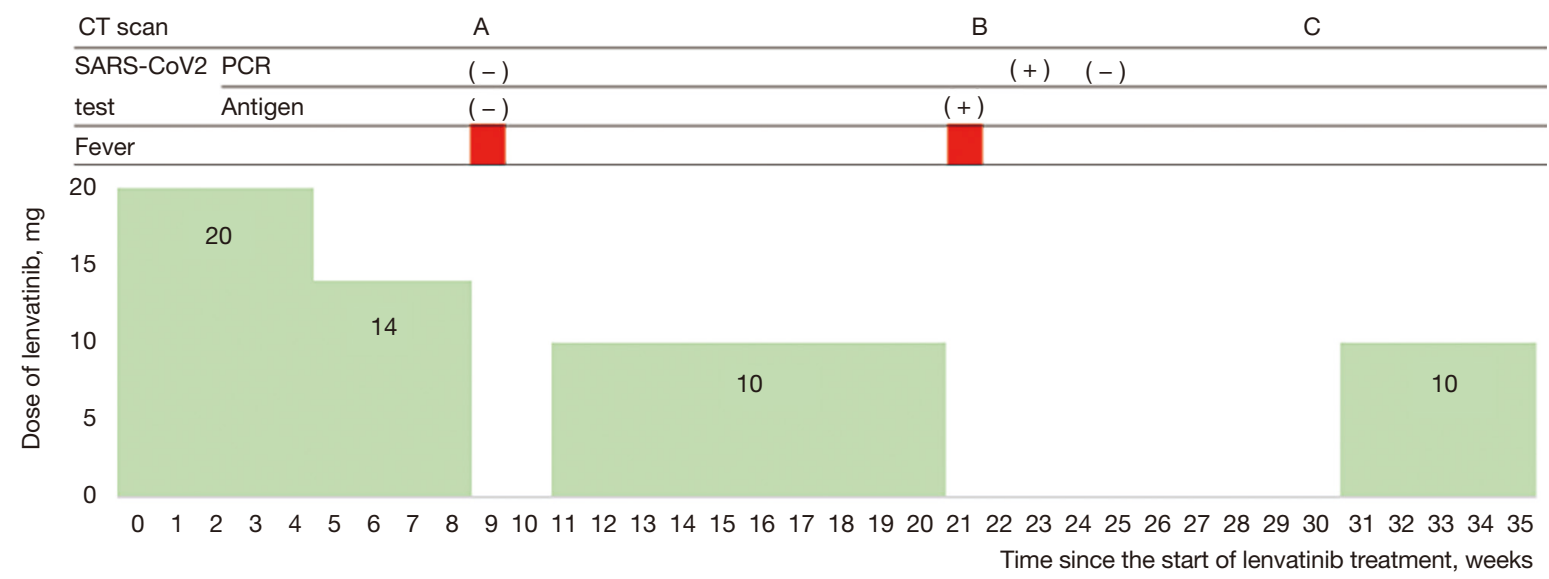

Figure 4 Graph showing the dose of lenvatinib and the time since treatment initiation. The timings of CT, viral tests, and fevers are depicted in the graph. The timings of CT A, B, and C match those shown in Figure 2. At 9 weeks after lenvatinib treatment initiation, the patient developed fever and pneumonia, which improved after the withdrawal of lenvatinib. At 21 weeks after lenvatinib treatment initiation, he developed fever again and tested positive for the SARS-CoV2. CT, computed tomography; SARS-CoV-2, severe acute respiratory syndrome coronavirus 2 .

Japanese authorities' criteria. Treatment with dexamethasone plus remdesivir and unfractionated heparin, which was used as a venous thromboembolism prophylaxis, was initiated.

On Day 2 of treatment, his body temperature normalized and the supplemental oxygen therapy was discontinued. He was discharged on Day 8 of hospitalization (Figure 3). The result of the SARS-CoV-2 RTPCR test performed on Day 11 of illness was positive. However, the test result was negative on Day 21 of illness. CT scan performed at 6 weeks of illness showed that the GGO in both sides of the lung had disappeared. At 10 weeks, pleural effusion and pulmonary metastases worsened and pulmonary cavitation remained. Owing to the progression of thyroid carcinoma, lenvatinib at a dose of $10 \mathrm{mg}$ was started (Figure 4).

All procedures performed in the study were in accordance with the ethical standards of the institutional research committee and with the Declaration of Helsinki (as revised in 2013). A written informed consent was obtained from the patient for the publication of this case report and accompanying images. A copy of the written consent is available for review by the editorial office of this journal. 


\section{Discussion}

Lenvatinib is a multi-tyrosine kinase inhibitor that suppresses signaling via the vascular endothelial growth factor receptors $1-3$, fibroblast growth factor receptors $1-4$, platelet-derived growth factor receptor alpha, and the RET and KIT proto-oncogene receptor tyrosine kinases. The primary antitumor effect of lenvatinib is exerted through the inhibition of angiogenesis $(8,9)$. Some studies have reported the occurrence of pulmonary cavitation and pneumothorax during lenvatinib treatment for pulmonary metastasis in patients with thyroid cancer $(3,4)$. In a retrospective cohort study of patients with thyroid cancer, $12 \%$ of a total of 83 patients with pulmonary metastasis developed cavitation during a treatment with tyrosine kinase inhibitors (10).

Angiogenesis inhibitors such as bevacizumab and pazopanib can also cause pulmonary cavitation and pneumothorax (11). The incidence rate of pneumothorax due to pazopanib treatment for metastatic soft tissue sarcomas has been reported to be $3.3-14 \%$ (12-14). Both the check-valve induced by tumor obstruction of airway and the pulmonary infarct caused by tumor embolism may lead to cavitation and pneumothorax (15).

COVID-19 is an infectious disease characterized primarily by pneumonia. Its most common finding on CT is GGO, typically with a peripheral and subpleural distribution. Later in the clinical course of the disease, the CT findings vary, and these include focal consolidation, a crazy paving pattern, a reverse halo sign, and a white lung appearance, which is an indicative of extensive GGO in the entire lung $(16,17)$. In some cases, pulmonary cavitation is associated with COVID-19. Cavitation may correlate with diffuse alveolar damage, intra-alveolar hemorrhage, and parenchymal cell necrosis (5). Moreover, it is associated with secondary complications such as hemoptysis and pneumothorax and with a poor prognosis (7). Secondary bacterial infection during COVID-19 treatment may also induce lung cavitation, which can be improved with antibacterial drugs (6).

In the present case, an indistinct shadow appeared in the patient's lungs around the consolidation after lenvatinib treatment initiation; afterward, it became a cavitary lesion. On the other hand, when the patient had COVID-19, multiple well-defined GGOs appeared on both sides of the lung. Although some reports have stated that lung metastasis leads to cavitation due to tumor necrosis, no tumor was detected in our patient at the site of cavitation.
Because the patient was not at a late stage of COVID-19 and showed improvements without having to use any antibacterial drugs, pulmonary cavitation might not have induced by COVID-19. The causes of pulmonary cavity include the presence of small lesions undetectable on CT or the effect of lenvatinib on angiogenesis. Pulmonary cavitation may occur at sites without lung metastases during lenvatinib treatment. As other differential diagnoses, the late stage of COVID-19 and secondary bacterial infection should also be considered during the COVID-19 pandemic.

\section{Acknowledgments}

The authors would like to thank Enago (https://www.enago. jp) for the English language review.

Funding: None.

\section{Footnote}

Reporting Checklist: The authors have completed the CARE reporting checklist. Available at https://apm.amegroups. com/article/view/10.21037/apm-21-2663/rc

Conflicts of Interest: All authors have completed the ICMJE uniform disclosure form (available at https://apm. amegroups.com/article/view/10.21037/apm-21-2663/coif). The authors have no conflicts of interest to declare.

Ethical Statement: The authors are accountable for all aspects of the work in ensuring that questions related to the accuracy or integrity of any part of the work are appropriately investigated and resolved. All procedures performed in the study were in accordance with the ethical standards of the institutional research committee and with the Declaration of Helsinki (as revised in 2013). A written informed consent was obtained from the patient for the publication of this case report and accompanying images. A copy of the written consent is available for review by the editorial office of this journal.

Open Access Statement: This is an Open Access article distributed in accordance with the Creative Commons Attribution-NonCommercial-NoDerivs 4.0 International License (CC BY-NC-ND 4.0), which permits the noncommercial replication and distribution of the article with the strict proviso that no changes or edits are made and the original work is properly cited (including links to both the 
formal publication through the relevant DOI and the license). See: https://creativecommons.org/licenses/by-nc-nd/4.0/.

\section{References}

1. Schlumberger $M$, Tahara $M$, Wirth LJ, et al. Lenvatinib versus placebo in radioiodine-refractory thyroid cancer. $\mathrm{N}$ Engl J Med 2015;372:621-30.

2. Iwasaki H, Toda S, Murayama D, et al. Relationship between adverse events associated with lenvatinib treatment for thyroid cancer and patient prognosis. Mol Clin Oncol 2021;14:28.

3. Kazzaz FI, Cabanillas ME, Bashoura L, et al. Bilateral spontaneous pneumothoraces in anaplastic thyroid cancer. Respir Med Case Rep 2019;26:197-9.

4. Yamazaki H, Iwasaki H, Yamashita T, et al. A Case of Pneumothorax after Treatment with Lenvatinib for Anaplastic Thyroid Cancer with Lung Metastasis. Case Rep Endocrinol 2018;2018:7875929.

5. Selvaraj V, Dapaah-Afriyie K. Lung cavitation due to COVID-19 pneumonia. BMJ Case Rep 2020;13:237245.

6. Ammar A, Drapé JL, Revel MP. Lung cavitation in COVID-19 pneumonia. Diagn Interv Imaging 2021;102:117-8.

7. Zoumot Z, Bonilla MF, Wahla AS, et al. Pulmonary cavitation: an under-recognized late complication of severe COVID-19 lung disease. BMC Pulm Med 2021;21:24.

8. Matsui J, Yamamoto Y, Funahashi Y, et al. E7080, a novel inhibitor that targets multiple kinases, has potent antitumor activities against stem cell factor producing human small cell lung cancer H146, based on angiogenesis inhibition. Int J Cancer 2008;122:664-71.

9. Matsui J, Funahashi Y, Uenaka T, et al. Multi-kinase

Cite this article as: Toda S, Matsui A, Yasukawa M, Murayama D, Iwasaki H. Pulmonary cavitation in a patient with coronavirus disease 2019 during lenvatinib treatment for thyroid carcinoma: a case report. Ann Palliat Med 2022;11(8):27512755. doi: 10.21037/apm-21-2663 inhibitor E7080 suppresses lymph node and lung metastases of human mammary breast tumor MDAMB-231 via inhibition of vascular endothelial growth factor-receptor (VEGF-R) 2 and VEGF-R3 kinase. Clin Cancer Res 2008;14:5459-65.

10. Datar S, Cabanillas M, Dadu R, et al. Pulmonary cavitation in patients with thyroid cancer receiving antiangiogenic agents. BMC Cancer 2020;20:1181.

11. Ozaki Y, Yoshimura A, Sawaki M, et al. Mechanisms and anatomical risk factors of pneumothorax after Bevacizumab use: A case report. World J Clin Oncol 2020;11:504-9.

12. Nakamura T, Matsumine A, Kawai A, et al. The clinical outcome of pazopanib treatment in Japanese patients with relapsed soft tissue sarcoma: A Japanese Musculoskeletal Oncology Group (JMOG) study. Cancer 2016;122:1408-16.

13. van der Graaf WT, Blay JY, Chawla SP, et al. Pazopanib for metastatic soft-tissue sarcoma (PALETTE): a randomised, double-blind, placebo-controlled phase 3 trial. Lancet 2012;379:1879-86.

14. Verschoor AJ, Gelderblom H. Pneumothorax as adverse event in patients with lung metastases of soft tissue sarcoma treated with pazopanib: a single reference centre case series. Clin Sarcoma Res 2014;4:14.

15. Çelik B, Sürücü ZP, Yılmaz V, et al. A Case Report of Secondary Simultaneous Bilateral Pneumothorax Due to Pazopanib Treatment. Turk Thorac J 2018;19:49-51.

16. Hani C, Trieu NH, Saab I, et al. COVID-19 pneumonia: A review of typical CT findings and differential diagnosis. Diagn Interv Imaging 2020;101:263-8.

17. Shi $H$, Han $X$, Jiang $N$, et al. Radiological findings from 81 patients with COVID-19 pneumonia in Wuhan, China: a descriptive study. Lancet Infect Dis 2020;20:425-34. 